\title{
ESTIMATING TORSION OF DIGITAL CURVES USING 3D IMAGE ANALYSIS
}

\author{
Christoph BlankenburG ${ }^{\bigotimes, 1,2,3}$, Christian Daul ${ }^{1,2}$ AND JOACHIM OHSER $^{3}$ \\ ${ }^{1}$ Université de Lorraine, CRAN, UMR 7039, 2 avenue de la Forêt de Haye, 54516 Vandouvre-lès-Nancy, \\ France; ${ }^{2}$ CNRS, CRAN, UMR 7039, 54516 Vandœuvre-lès-Nancy, France; ${ }^{3}$ Univ. Appl. Sci. Darmstadt, Dept. \\ Math \& Nat. Sci., Schöfferstr. 3, 64295 Darmstadt, Germany \\ e-mail: christoph.blankenburg@univ-lorraine.fr, christian.daul@univ-lorraine.fr, jo@h-da.de \\ (Received November 2, 2015; revised March 1, 2016; accepted March 7, 2016)
}

\begin{abstract}
Curvature and torsion of three-dimensional curves are important quantities in fields like material science or biomedical engineering. Torsion has an exact definition in the continuous domain. However, in the discrete case most of the existing torsion evaluation methods lead to inaccurate values, especially for low resolution data. In this contribution we use the discrete points of space curves to determine the Fourier series coefficients which allow for representing the underlying continuous curve with Cesàro's mean. This representation of the curve suits for the estimation of curvature and torsion values with their classical continuous definition. In comparison with the literature, one major advantage of this approach is that no a priori knowledge about the shape of the cyclic curve parts approximating the discrete curves is required. Synthetic data, i.e., curves with known curvature and torsion, are used to quantify the inherent algorithm accuracy for torsion and curvature estimation. The algorithm is also tested on tomographic data of fiber structures and open foams, where discrete curves are extracted from the pore spaces.
\end{abstract}

Keywords: discrete geometry, integral of torsion, skeleton line.

\section{INTRODUCTION}

The curvature of a space curve measures the rate of change of the tangent when moving along the curve, while the torsion (also referred to as the second curvature) is defined as the rate of change of the curve's osculating plane. Both quantities have clear differential geometric meanings; the curvature is a second-derivative quantity and the torsion forms a third-derivative quantity.

First of all, we recall that in materials sciences it is well known for a long time that the torsion of dislocation lines in a crystal is closely related to the density of inflections that are optically discernible in transmission electron microscopy (Rhines, 1977). This fundamental perception traces back to Fullman (1953). Since this time there was published a continuously growing number of articles on the investigation of the impact of torsion of dislocations on macroscopic tensile strength (Zhao et al., 2012) and, vice versa, of the torsion tensor of the crystal on dislocation motion. Furthermore, the torsion of paths through the pore space is an important quantity of porous media with respect to filter efficiency and deposition rates of two-phase flow. As an example we consider porous beryllium used in the helium cooled pebble bed (HCPB) blankets investigated in the framework of the European Fusion Technology Programme. The long-term tritium and helium accumulation in HCPB blankets is crucial for the reliable and safe operation of fusion reactors. It depends on the percolation, the curvature as well as the torsion of the pore space (Moeslang et al., 2009; Pieritz et al., 2011; Ohser et al., 2012). As pointed out in Bae et al. (2013), low torsion can have a considerable impact on carrier mobility and, thus, on cell charging and discharging of batteries with porous electrodes. Finally, we remark that mechanical properties like strength and stiffness of fiber reinforced materials depend on the torsion of the fibers' center lines.

A further topic is the investigation of the spacetime trajectories of particles moving in magnetic or gravitational fields. The geometric properties of particle trajectories in turbulent two-phase flows are investigated in Scagliarini (2011). It is shown in this article that Lagrangian tracers can have significant curvature and torsion (depending on smallscale turbulence features and the velocity magnitude). Similar trajectory studies are of interest in biology. For example, the velocity, curvature and torsion of threedimensional (3D) trajectories of organisms is studied in Crenshaw et al. (2000). Indeed, as has been pointed out, the torsion of trajectories of freely swimming organisms is an important characteristic for biological activity.

Applications in medicine mainly concentrate on the geometric characterization of arteries. The 
relationship between the torsion of the internal carotid artery in human infants and the brain temperature is studied in Meng et al. (2008). A large absolute torsion correlates with the occurrence of vascular pathologies. Similar investigations of the torsion based on tomographic data are made for the coronary artery (Pao et al., 1992; Puentes et al., 1998; Medina et al., 2004; Zhenga and Qib, 2011; Strandmark et al., 2013) and for the superficial femoral arteries (Wood et al., 2006). The methodology in medical applications differs from that in other fields of application. The aim in medicine is to characterize an individual object (e.g., an artery by its torsion), while in materials science and two-phase flow (macroscopically homogeneous) systems of objects (e.g., the trajectories of particles) are investigated.

There are various approaches of estimating local torsion from 3D images, which differ in their strategies of handling the discretization effects. The approach suggested in Kehtarnavaz and de Figueiredo (1988) is based on smoothing splines fitted to discrete curves, where the torsion is estimated from the splines. A similar concept is used in Medina et al. (2004) and Lewinger et al. (2005), where models of continuous curves are fitted to discrete ones by least-squares methods, and the continuous curve representations are again used for torsion assessment. In Pao et al. (1992) and Crenshaw et al. (2000), cubic splines are fitted to the positions of anatomic landmarks and organisms, respectively. Mokhtarian (1997) follows a multiscale description of curve shape and applies anisotropic Gaussian smoothing to attenuate discretization effects on torsion estimations. It is obvious to speed up smoothing by Fourier techniques, which leads to fast torsion estimation (Medina et al., 2004). This approach is very skillful since the derivatives of the space curve necessary for the calculation of the torsion can also be computed via the inverse space (simultaneously with the smoothing). The core of the algorithmic approach suggested by Nguyen and Debled-Rennesson (2008; 2009) is a decomposition of discrete curves into socalled 'blurred' segments, which is fundamentally the same as smoothing. In Blankenburg et al. (2015) discrete versions of differential geometric formulas for the curvature and torsion are applied to the characterization of particle motion in porous media during a filtration process.

A path through the percolating pore space of a 3D image of a porous medium or the skeleton of a fiber can be seen as a discrete curve consisting of pixels that are connected with respect to a chosen adjacency system (also known as the pixel neighborhood). Possible choices are the 6-, the 14.1-, the 14.2-, or the 26adjacency (Ohser et al., 2009; Ohser and Schladitz,
2009). The curve length can be estimated from local pixel configurations using the technique described in Ohser et al. (2012). This technique is based on a discretization of the Crofton intersection formula for the integral of the mean curvature (Schneider, 1993), cf. also Coeurjolly et al. (2001); Coeurjolly and Svensson (2003) where an alternative approach is presented. The backbone of a corresponding algorithm is simply the determination of the numbers of $2 \times$ $2 \times 2$-pixel configurations of a binary image and the computation of a weighted sum of these numbers. The simplicity of this method is due to the fact that the integral of the mean curvature is an additive functional. However, the curvature integral and the torsion integral of space curves are not additive and, therefore, it cannot be expected that they can be estimated from local knowledge, only.

One should keep in mind that in image analysis the term "tortuosity" (sometimes called "geometric tortuosity") is widely used for the ratio of the (Euclidean) distance between the end points of a curve and the curve length, where the curve length is equivalent to the geodesic distance. This can be confusing, because the terms "torsion" and "tortuosity" are often used synonymously. However, the tortuosity (in the above setting) fundamentally differs from torsion. In Patasius et al. (2007) the tortuosity is applied to the characterization of blood vessels. This paper also gives a survey on various variants of the definition of tortuosity known from the literature. In particular, the tortuosity depends on how the geodesic distance is measured (Grisan et al., 2003). This problem is addressed in detail in Chen-Wiegart et al. (2014), where the backbone of application are porous $\mathrm{LiCoO}_{2}$ cathode electrodes of lithium ion batteries. A similar approach is used in Gommes et al. (2009) for the characterization of porous ethylene vinyl acetate. In Peyrega and Jeulin (2013) the tortuosity is estimated from $\mu \mathrm{CT}$ images of Thermisorel structure (a fiber material applied for acoustic absorption), where special attention is paid to the reconstruction of geodesic paths. We also refer to the paper Gaiselmann et al. (2013) where the tortuosity of the fibers of a non-woven material was computed.

In the present paper we give a fast algorithm for the computation of the torsion from discrete curves in 3D images, where the discretization is obtained from the sampling of a continuous parametric function on the corresponding 3D lattice. Our approach is based on the fitting of a partial sum of a Fourier series to the discrete curve and the torsion estimation of the curve from the partial sum. The idea behind is that one can separate low frequencies belonging to the parametric function and higher frequencies resulting from discretization. 
Unfortunately, the periodicity implicitly induced by the Fourier series approach usually leads to a discontinuity in the parametric function that can significantly influence the magnitudes of the high frequencies. On the other hand, discretization modifies low frequencies as well, even for a small pixel size. To overcome these problems, we make use of Fejér's theorem (Zygmund, 1988) and estimate the torsion from Cesàro's mean instead from a partial sum.

As an application we consider the torsion of possible cell paths through the pore spaces of a partially open foam or a fiber system. These porous media have a specifically activated inner surface which makes them useful in cell chromatography. The torsion of such cell paths is highly important for the efficiency of chromatographic filters. It influences the deposition rates of cells at the inner surface.

The paper is organized as follows: First, we give a short introduction to some differential geometric foundations. In the next section we introduce the Jordan discretization, which is closely related to a sampling of the curve on the 3D lattice. Then the Fourier series approach is described, where the consequences of the periodicity assumption as well as the influence of discretization on accuracy of estimation is studied in detail. Finally, this algorithm is tested using synthetic data as well as tomographic images of porous media.

\section{CURVATURE AND TORSION OF SPACE CURVES}

A space curve $C$ may be given by

$$
C=\left\{f(t): t_{0} \leq t \leq t_{1}\right\},
$$

where $f(t)$ is a rectifiable parametric function, $f$ : $\mathbb{R} \mapsto \mathbb{R}^{3}$. Assuming that the derivatives $\dot{f}$ and $\ddot{f}$ are continuous, the length $\mathscr{L}(C)$ of the curve $C$ and the (local) curvature $\kappa$ exist,

$$
\mathscr{L}(C)=\int_{C} d x=\int_{t_{0}}^{t_{1}}\|\dot{f}\| \mathrm{d} t, \quad \kappa=\frac{\|\dot{f} \times \ddot{f}\|}{\|\dot{f}\|^{3}},
$$

(Spivak, 1979), where $\int_{C} \mathrm{~d} x$ means the integration on $C$ with respect to the length measure, and $\times$ denotes the cross product. In the following the norm $\|\dot{f}\|$ of the first derivative will be called the length density. Furthermore, if $\kappa>0$ and $\dddot{f}$ is continuous, then the local torsion $\tau$ is well defined,

$$
\tau=\frac{(\dot{f} \times \ddot{f}) \cdot \dddot{f}}{\|\dot{f} \times \ddot{f}\|^{2}},
$$

(Spivak, 1979). Notice that unlike the curvature the torsion is a signed quantity. The length $\mathscr{L}(C)$, the integral of curvature $\mathscr{K}(C)=\int_{C} \kappa \mathrm{d} x$ and the integral of torsion $\mathscr{T}(C)=\int_{C} \tau \mathrm{d} x$ are basic characteristics of C.

The unit of $\kappa$ and $\tau$ is $\mathrm{m}^{-1}$, and thus, their integrals $\mathscr{K}$ resp. $\mathscr{T}$ are unscaled, i. e.

$$
\mathscr{K}(a C)=\mathscr{K}(C), \quad \mathscr{T}(a C)=\mathscr{T}(C),
$$

for all $a>0$. Furthermore, $\mathscr{K}$ and $\mathscr{T}$ are invariant with respect to translations and rotations of $C$. Finally, we remark that the reflection of $C$ at a plane changes the sign of $\mathscr{T}(C)$.

Notice that if the curve $C$ does not have multiple points, then its length is the geodesic distance between the two end points $f\left(t_{0}\right)$ and $f\left(t_{1}\right)$. The Euclidean distance is $\left\|f\left(t_{1}\right)-f\left(t_{0}\right)\right\|$, and the tortuosity of $C$ is usually defined as the ratio

$$
\frac{\mathscr{L}(C)-\left\|f\left(t_{1}\right)-f\left(t_{0}\right)\right\|}{\mathscr{L}(C)},
$$

which takes values between 0 and 1 . This ratio can be 1 (maximum tortuosity) even if the torsion $\tau$ is zero almost everywhere on $C$.

Throughout this paper we consider a class of space curves given by

$$
f(t)=r\left(\begin{array}{c}
\cos t \\
\sin t \\
g(t)
\end{array}\right), \quad t \in[0,2 \pi), r>0,
$$

where $r$ is a scaling factor and the function $g: \mathbb{R} \mapsto \mathbb{R}$ is continuous and threefold differentiable on $[0,2 \pi)$.

Example 1. The probably most popular example of a space curve belonging to the class defined by Eq. 1 is the helix. Using $g(t)=c t$ one gets a helix with the pitch $2 \pi r c$ and the length $\mathscr{L}(C)=2 \pi r \sqrt{1+c^{2}}$. One can easily verify that curvature and torsion are independent of $t$,

$$
\kappa=\frac{1}{r\left(1+c^{2}\right)}, \quad \tau=\frac{c}{r\left(1+c^{2}\right)} .
$$

Both, the integral of curvature $\mathscr{K}(C)=2 \pi / \sqrt{1+c^{2}}$ and the integral of torsion $\mathscr{T}(C)=2 \pi c / \sqrt{1+c^{2}}$ are independent of $r$.

Example 2. Fig. 1 shows the curve $C$ for $g(t)=c \sin ^{2} t$. The periodicity of $g$ implies that $C$ is cyclic. The parametric function $f$ is infinitely differentiable and one obtains

$$
\|\dot{f}(t)\|=\frac{r}{\sqrt{2}} \sqrt{2+c^{2}(1-\cos 4 t)} .
$$


Hence, the curve length is

$$
\mathscr{L}(C)=2 r\left(E\left(-c^{2}\right)+\sqrt{1+c^{2}} E\left(\frac{c^{2}}{1+c^{2}}\right)\right),
$$

where $E$ is the complete elliptic integral of the 2nd kind. Here, we use the setting

$$
E(x)=\int_{0}^{\frac{\pi}{2}} \sqrt{1-x \sin ^{2} \vartheta} \mathrm{d} \vartheta, \quad x \leq 1 .
$$

The local curvature can be expressed as

$$
\kappa(t)=\frac{2}{r} \sqrt{\frac{2+c^{2}(5+3 \cos 4 t)}{\left(2+c^{2}(1-\cos 4 t)\right)^{3}}},
$$

and the integral of curvature is given by the integral

$$
\mathscr{K}(C)=\sqrt{2} \int_{0}^{2 \pi} \frac{\sqrt{2+c^{2}(5+3 \cos 4 t)}}{2+c^{2}(1-\cos 4 t)} \mathrm{d} t,
$$

which can be computed numerically, only. Finally, the local torsion can be calculated as

$$
\tau(t)=-\frac{6 c \sin 2 t}{r\left(2+c^{2}(5+3 \cos 4 t)\right)},
$$

and its integration yields

$$
\int_{0}^{\pi / 2} \tau(t) \| \dot{f}(t) \mathrm{d} t=\arctan c-2 \arctan 2 c .
$$

which converges to $-\frac{\pi}{2}$ as $c \rightarrow \infty$. Obviously, the integral of torsion vanishes, $\mathscr{T}(C)=0$.

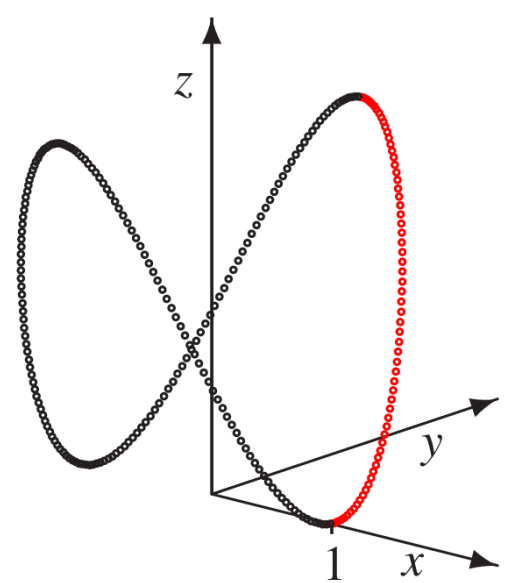

Fig. 1. The curve $C$ of the parametric function $f(t)$ with $g(t)=c \sin ^{2}(t), r=1$ and $c=2$. The red part is for $0 \leq t \leq \frac{\pi}{2}$.

\section{DISCRETE CURVES}

In the context of image analysis we consider a discretization of the curve $C$ induced by sampling on lattice points. Let $\mathbb{Z}^{3}$ be the homogeneous $3 \mathrm{D}$ lattice of the lattice spacing 1 (which is identical with the pixel size), where $\mathbb{Z}$ is the set of integers and $[0,1)^{3}$ is the half-open unit cell. The sampling $C_{\square}$ of $C$ on the lattice $\mathbb{Z}^{3}$ is the set of all pixels $x \in \mathbb{Z}^{3}$ with the property that $C$ hits the shifted cell $[0,1)^{3}+x$,

$$
C_{\square}=\left\{x \in \mathbb{Z}^{3}: C \cap\left([0,1)^{3}+x\right) \neq \emptyset\right\} .
$$

The union

$$
\bigcup_{x \in C \square}\left([0,1)^{3}+x\right)
$$

of the lattice cells hitting $C$ is well known as the outer Jordan discretization of $C$. Under certain assumptions for the shape of $C$, the sampling $C_{\square}$ is a discrete curve with respect to the 6-adjacency of the pixels. More precisely, we suppose that $C$ may have a shape such that $C_{\square}$ is a skeleton line with respect to the 6adjacency; i.e., $C_{\square}$ is the skeleton of itself. A necessary condition for that is that the curvature of $C$ must be bounded.

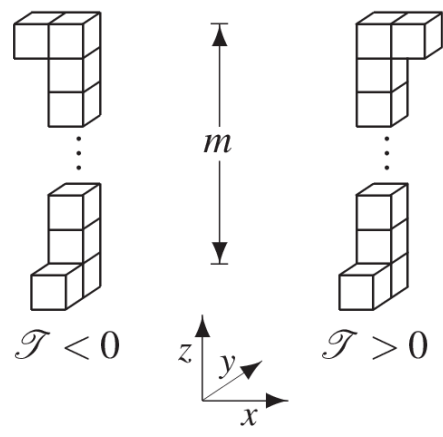

Fig. 2. Outer Jordan discretizations with $m+3$ pixels of a curve piece.
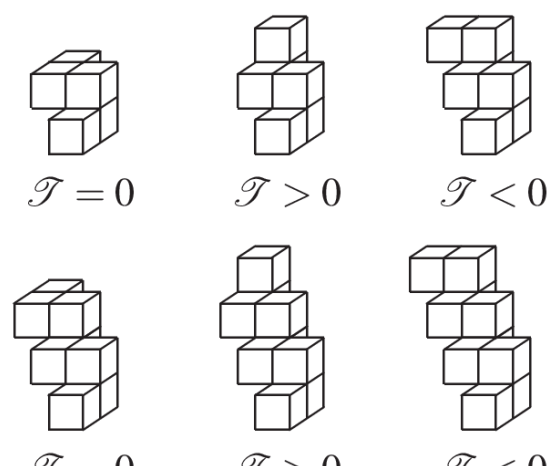

$$
\mathscr{T}=0
$$

$\mathscr{T}>0$

$$
\mathscr{T}<0
$$

Fig. 3. A sequence of outer Jordan discretizations with growing pixel number. 
Examples of outer Jordan discretizations of curve pieces are shown in Figs. 2 and 3. Using the righthand rule one can check whether the integral of torsion $\mathscr{T}$ is negative, zero or positive. Such a decision is based on the assumption that the curve pieces behind the discretizations are sufficiently smooth with almost everywhere existing local curvature $\kappa$. For example, one can fit the curve given in Example 2 for $0 \leq t \leq$ $\pi / 2$ (red marked part in Fig. 1) to the outer Jordan discretization shown in Fig. 2 (left). A possible choice of the parameters is $r=1$ and $c=m$. Then from Eq. 2 it immediately follows that $\mathscr{T} \approx-\pi / 2$ for a sufficiently large pixel number $m$. Analogously, one could fit pieces of sufficiently smooth continuous curves to other discrete curves. Nonetheless, fitting continuous curves to discrete ones could be a challenging problem. This is demonstrated in Fig. 3, where the sign of $\mathscr{T}$ estimated from the right-hand rule switches when successively appending pixels.

We remark that improving the lateral resolution, i.e., reducing the pixel size, is equivalent to increasing the scaling factor $r$. More precisely, the sampling of $f$ on a lattice $p \mathbb{Z}^{3}$ with the pixel size $p>0$ is the same as the sampling of $\frac{1}{p} f$ on $\mathbb{Z}^{3}$.

\section{FOURIER APPROXIMATION}

In this section we present a Fourier approach for estimating curvature and torsion from discrete curves $C_{\square}$. The problem is to fit a Fourier series to $C_{\square}$. First we assume that the parametric function $f: \mathbb{R} \mapsto$ $\mathbb{R}^{3}$ is periodic with the period length $a$, i.e., the corresponding curve $C$ is cyclic. If $f$ is Lebesgue integrable on $(0, a)$, its Fourier coefficients

$$
c_{\ell}=\frac{1}{a} \int_{0}^{a} f(t) \mathrm{e}^{-\frac{2 \pi i \ell t}{a}} \mathrm{~d} t, \quad \ell \in \mathbb{Z},
$$

exist, where $c_{\ell}=\left(c_{\ell, 1}, c_{\ell, 2}, c_{\ell, 3}\right) \in \mathbb{C}^{3}$ and $\mathbb{C}$ is the space of complex numbers. Furthermore,

$$
s_{m}(t)=\sum_{\ell=-m}^{m} c_{\ell} \mathrm{e}^{\frac{2 \pi i \ell t}{a}}, \quad m=0,1, \ldots
$$

is the $m$-th partial sum of the Fourier series. We remark that the derivatives $\dot{s}_{m}, \ddot{s}_{m}, \dddot{s}_{m}$ and the functions $\left\|\dot{s}_{m}\right\|^{2}$, $\dot{s}_{m} \times \ddot{s}_{m},\left\|\dot{s}_{m} \times \ddot{s}_{m}\right\|^{2}$ and $\left(\dot{s}_{m} \times \ddot{s}_{m}\right) \cdot \dddot{s}_{m}$ are partial sums of Fourier series, too. This motivates us to fit a partial sum to a sampling of a space curve and to estimate the curvature and torsion of the curve from the partial sum.

Example 3. In order to discuss the problems that can arise in our Fourier series approach, we consider the helix introduced in Example 1. Its nonzero Fourier coefficients are $c_{-1,1}=c_{1,1}=1 / 2, c_{2,-1}=-i / 2$, $c_{2,1}=i / 2, c_{0,3}=\pi$ and $c_{k, 3}=-i / k$ for $k \neq 0$. The Fourier series is convergent, i.e., $s_{m}(t) \rightarrow f(t)$ as $m \rightarrow$ $\infty$. The Parseval identity yields

$$
\frac{1}{2 \pi} \int_{0}^{2 \pi}\|f(t)\|^{2} \mathrm{~d} t=\sum_{k=-\infty}^{\infty}\left\|c_{k}\right\|^{2}=r^{2}\left(1+\frac{4 \pi}{3} c^{2}\right) .
$$

However, the periodicity assumption implies a discontinuity of $f(t)$ at $t=0$ and, therefore, the first derivative of the Fourier series is divergent. In particular, we get

$$
\lim _{m \rightarrow \infty} \frac{1}{2 \pi} \int_{0}^{2 \pi}\left\|\dot{s}_{m}(t)\right\|^{2} \mathrm{~d} t=\lim _{m \rightarrow \infty} \sum_{k=-m}^{m}\left\|i k c_{k}\right\|^{2}=\infty .
$$

As a consequence, the length of the space curve given by the Fourier series of the helix with $c \neq 0$ does not exist. Analogously, the $L^{2}$-norms of the higher-order derivatives of $s_{m}$ are divergent, and thus, the integral of curvature $\mathscr{K}$ and the integral of torsion $\mathscr{T}$ of $s_{m}$ diverge, too.

From the previous example we have seen, that the higher-order derivatives of $s_{m}$ do not necessarily converge to the higher-order derivatives of $f$ even if $s_{m}(t) \rightarrow f(t)$ as $m \rightarrow \infty$. Thus, instead of the partial sum $s_{m}$ we consider the corresponding Cesàro mean $\sigma_{m}$, which is simply the arithmetic mean of the first partial sums,

$$
\sigma_{m}(t)=\frac{1}{m+1} \sum_{\ell=0}^{m} s_{\ell}(t) .
$$

Fejér's theorem states that if the left and right limits $f(t \mp 0)$ exist, then

$$
\lim _{m \rightarrow \infty} \sigma_{m}(t)=\frac{f(t+0)+f(t-0)}{2}
$$

for all $t \in[0, a)$ (Zygmund, 1988). For this reason, the Fejér summability of a Fourier series is even more important than its convergence.

Let now $C_{\square}$ be a sampling of the curve $C$ of a parametric function $f(t)$ which is assumed to be periodic on $[0, a)$. The sampling $C_{\square}$ may consist of $n$ pixels with the positions $f_{0}, \ldots, f_{n-1}$. The $m$-th partial sum $s_{m}(t)$ of a Fourier series can be fitted to the sampling $C_{\square}$ using the approximation $c_{\ell} \approx \tilde{f}_{\ell}$, where $\left(\tilde{f}_{0}, \ldots, \tilde{f}_{n-1}\right)$ denotes the discrete Fourier transform of the vector $\left(f_{0}, \ldots, f_{n-1}\right)$ with the setting

$$
\tilde{f}_{\ell}=\frac{1}{n} \sum_{k=0}^{n-1} f_{k} \mathrm{e}^{-\frac{2 \pi i k \ell}{n}}, \quad \ell=0, \ldots, n-1 .
$$


With regard to an explicit presentation of the corresponding Cesàro mean, the partial sums $s_{m}^{\square}(t)$ of the discrete curve $C_{\square}$ are now given by

$$
s_{m}^{\square}(t)=\tilde{f}_{0}+\sum_{\ell=1}^{m} \tilde{f}_{\ell} \mathrm{e}^{\frac{2 \pi i \ell t}{a}}+\sum_{\ell=n-m}^{n-1} \tilde{f}_{\ell} \mathrm{e}^{-\frac{2 \pi i \ell t}{a}}
$$

for $m \leq n / 2$ (Fourier approximation). Notice that the pixel positions $f_{k}$ are points on continuous and infinitely differentiable parametric function $s_{n / 2}^{\square}(t)$, i.e., pieces of the curve of $s_{n / 2}^{\square}(t)$ are interpolating between the $f_{k}$ (Fourier interpolation). Now Cesàro's mean $\sigma_{m}^{\square}(t)$ of the $s_{\ell}^{\square}(t), \ell=0, \ldots, m$, can be written as

$$
\begin{aligned}
\sigma_{m}^{\square}(t)=\tilde{f}_{0} & +\sum_{\ell=1}^{m} \frac{m+1-\ell}{m+1} \tilde{f}_{\ell} \mathrm{e}^{\frac{2 \pi i \ell t}{a}} \\
& +\sum_{\ell=n-m}^{n-1} \frac{\ell+m-n+1}{m+1} \tilde{f}_{\ell} \mathrm{e}^{-\frac{2 \pi i \ell t}{a}}
\end{aligned}
$$

From Fejér's theorem it follows that in the case of a continuous parametric function $f$ the $\sigma_{m}^{\square}(t)$ converges uniformly to $f(t)$ on $[0, a)$ for increasing lateral resolution (i.e. for decreasing pixel size) and as $m \rightarrow$ $\infty$. The function $\sigma_{m}^{\square}$ can be seen as a reconstruction of $f$ from $C_{\square}$. Furthermore, Cesàro's mean involves a smoothing that suppresses discretization noise, where the power of smoothing can be controlled by the choice of the parameter $m$. As a consequence, estimates of the local curvature and torsion from $\sigma_{m}^{\square}(t)$ usually have much smaller errors than those from the partial sums $s_{m}^{\square}(t)$, see Figs. 4 and 5 for examples. Moreover, even if the curvature of $s_{m}^{\square}(t)$ does not converge to the true curvature $\kappa(t)$, the curvature of $\sigma_{m}^{\square}(t)$ can pointwisely converge to $\kappa(t)$ for increasing lateral resolution and as $m \rightarrow \infty$. This holds, e.g.,, for the curve introduced in Example 2. Analogously, one can find examples of discrete curves with a divergent torsion of $s_{m}^{\square}(t)$ but with a convergent torsion of $\sigma_{m}^{\square}(t)$.

An extreme example is the periodically extended helix having a strong discontinuity at $t=0$. The results of curvature and torsion estimation are shown in Fig. 4. The frequencies resulting from the discontinuity are modulated with those caused by the sampling of the curve on the lattice. Clearly, the errors are dramatically large, but one can also see that in general the errors of the local curvature and torsion estimated form Cesàro's mean are much smaller than those from the corresponding partial sum. It turns out that for large $r$ the relative deviations from the true curvature and torsion are widely independent of $r$. This means that the estimation errors mainly result from the discontinuity of $f$ at $t=0$.
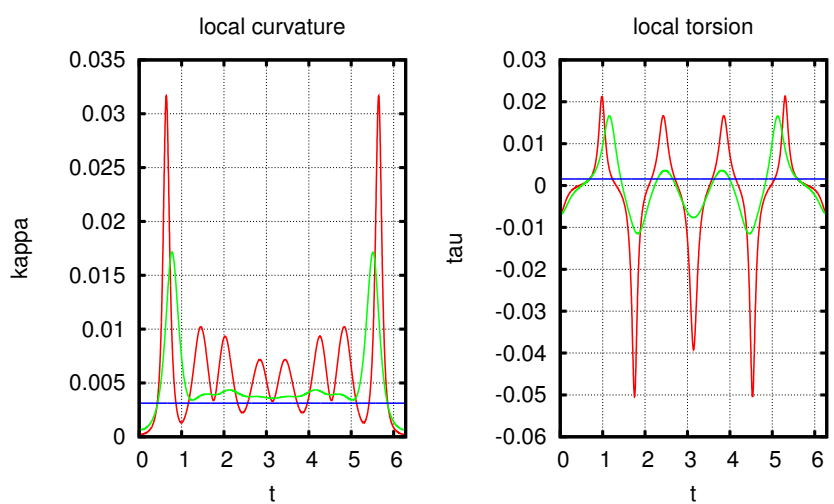

Fig. 4. Estimates of the local curvature $\kappa(t)$ (left) and the local torsion $\tau(t)$ (right) from the partial sum $s_{4}^{\square}(t)$ (red) and the Cesàro's mean $\sigma_{4}^{\square}(t)$ (green) of a sampling of the helix with the parameters $r=256$ and $c=0.5$. The blue lines depict the theoretical values.
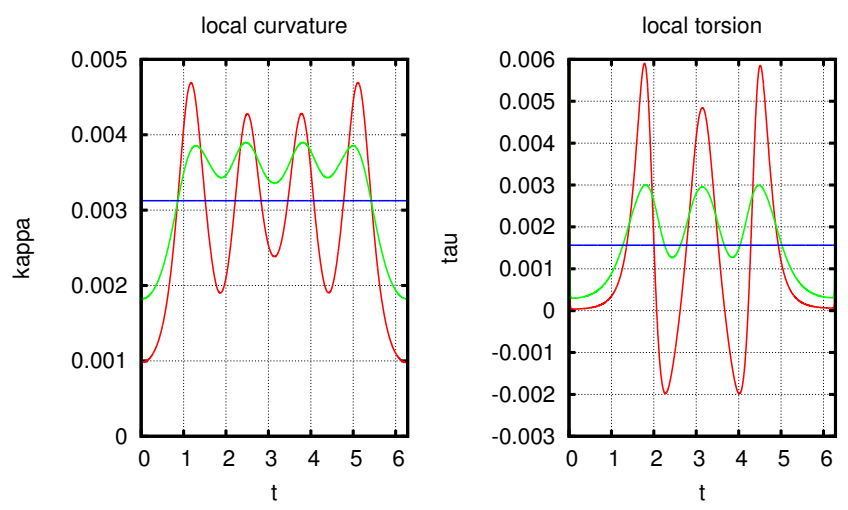

Fig. 5. Estimates of the local curvature $\kappa(t)$ (left) and the local torsion $\tau(t)$ (right) under reflection condition. The estimates are based on the partial sum $s_{8}^{\square}(t)$ (red) and Cesàro's mean $\sigma_{8}^{\square}(t)$ (green) of a sampling of the helix with the parameters $r=256$ and $c=0.5$. The blue lines depict the theoretical values.

Reflection of $f$ at $t=0$ removes the discontinuity. An appropriate reflection condition can be involved into the estimation procedure when replacing the sampling $\left(f_{0}, \ldots, f_{n-1}\right)$ with $\left(f_{0}, \ldots, f_{n-1}, f_{n-1}, \ldots, f_{0}\right)$ where now the pixel number is $2 n$. The smoothing parameter $m$ has to be adapted to the increased pixel number; instead of $m$ we use $2 \mathrm{~m}$. The effect of involving the reflection condition is shown in Fig. 5. The reduction of the estimation errors is considerable. The maximum curvature and torsion errors drop down from 0.015 and 0.016 (Fig. 4) to 0.001 and 0.0015 (Fig. 5), respectively. The errors are diminished by about one order of magnitude. 


\section{DISCRETIZATION EFFECTS}

In this section we study the influence of discretization on torsion estimation. To avoid discontinuity effects on estimation errors, we chose cyclic curves $C$ with everywhere continuous torsion. Furthermore, we want to make our investigations independent of the location of $C$ with respect to the lattice $\mathbb{Z}^{3}$. Thus, the discrete curves $C_{\square}$ are assumed to be samplings of independent and isotropic uniformly random (IUR) rotations of $C$ (Coleman and Pritchett, 1990) and, moreover, a random offset is added which is uniformly distributed on the lattice cell $[0,1)^{3}$. As a consequence, torsion estimates depend on $f$, the scaling and the smoothing parameter $m$, only. The mean deviation,

$$
\delta_{m}=\sqrt{\frac{1}{2 \pi n} \int_{0}^{2 \pi} \sum_{i=1}^{n}\left(\tau_{m, i}^{\square}(t)-\tau(t)\right)^{2} \mathrm{~d} t},
$$

of torsion estimates $\tau_{m, i}^{\square}(t), i=1, \ldots, n$, from the true torsion $\tau(t)$ quantifies the overall error.

Example 4. Let $f$ be the model of a curve given in Example 2. This model is flexible enough such that various curve shapes can be simulated by modifying the model parameters $r$ and $c$. In particular, $r$ and $c$ can be chosen such that the slope $\dot{\tau}(t)$ of the torsion strongly varies. Fig. 6 shows the results of a simulation study with the parameters $r=32, c=0.5$ and $m=4$. The average as well as the pointwise confidence band $(C B)$ for the local torsion were computed, i.e., for the outer Jordan discretizations of 256 IUR rotations of the curve $f_{2}(t)$ we have estimated the local torsion based on Cesàro's mean, and from these estimates we have pointwisely computed the average and the $C B$. Notice that it is sufficient to plot the curves for $0 \leq t<\pi$, since the function $\tau$ is odd. The overall error is $\delta_{4}=$ $6.961 \cdot 10^{-3}$. Related to the maximum true torsion of $37.5 \cdot 10^{-3}$ this is an error of about $18 \%$. Some further results are given in Table 1.

Clearly, the overall error decreases with an increasing scaling factor $r$ or, equivalently, with an increasing lateral resolution. Moreover, for $r>4$ the overall error becomes larger with increasing $c$. The last result is due to Cesàro's averaging which leads to a larger estimation bias in case of functions $\tau(t)$ with stronger slopes. Another aspect is that the scattering of pixels of $C_{\square}$ along $f$ depends on the tangent direction of $f, c f$. Figs. 2 and 3. The tangential change as well as the scattering keep their long-range behavior for large $r$. As a consequence, one cannot expect that the contribution of discretization to the overall error will completely vanish for increasing lateral resolution.
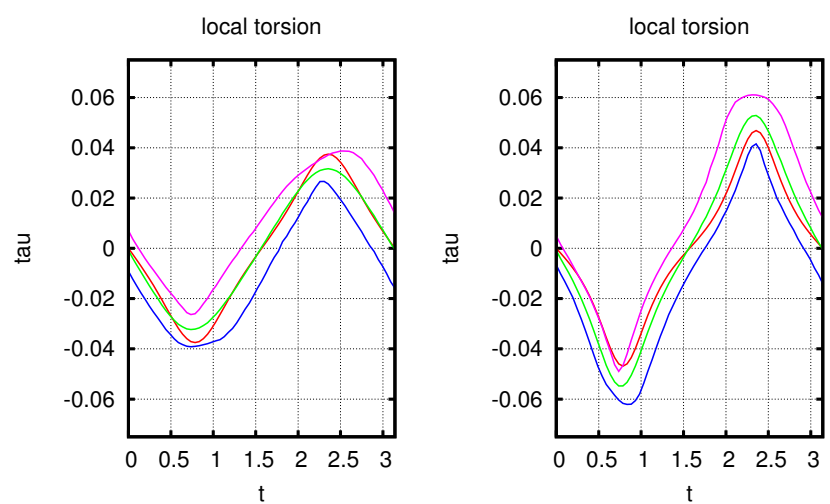

Fig. 6. Errors of estimation of the local torsion $\tau(t)$ from 1024 samplings of IUR rotations of the curve introduced in Example 2 with the parameters $r=32$ and $c=0.5$ (left) resp. $c=1.0$ (right). The estimation is based on Cesàro's mean $\sigma_{4}^{\square}(t)$ : theoretical torsion (red), the average over all samplings (green), the 5\%bound (blue) and the 95\%-bound (magenta) of the pointwise $C B$.

Table 1. Numerical values of the overall error $\delta_{4}$ of torsion estimation from 1024 samplings of IUR rotations of the curve introduced in Example 2.

\begin{tabular}{rccc}
\hline$r$ & $c=0.5$ & $c=1.0$ & $c=1.5$ \\
\hline 4 & 0.291455 & 0.255231 & 0.219882 \\
8 & 0.062333 & 0.068155 & 0.068929 \\
16 & 0.015936 & 0.023894 & 0.027741 \\
32 & 0.006961 & 0.010358 & 0.012785 \\
64 & 0.003983 & 0.004916 & 0.006186 \\
\hline
\end{tabular}

\section{APPLICATION}

The isolation of cells from body fluids or liquid culture media is a common procedure used in clinical diagnosis, molecular biology and other life sciences. Techniques like fluorescence assisted cell sorting, magnetic bead assisted cell sorting and micro-fluid separation are laboratory methods applicable for small amounts of cells and sample volumes, only. Centrifugation is the technique mostly used for separation of cells from human blood circulatory systems or for harvesting cells from industrial incubators. Disadvantages of centrifugation are the loss of patient body fluid in therapy (e.g., in healthy and tumoral blood cells in case of leukemia) and the high investment and maintenance costs in industrial applications. It is well known that cells can be immobilized out of a micro-fluidic stream by surface-bound selective ligands. An appropriate device for cell isolation from larger volumes would 
be a flow-through-cartridge filled by a porous medium, where the target cells are specifically interacting with the inner surface. Therefore, modern development of technical cell separation is based on alternative approaches such as separation of target cells using specific interaction with the inner surface of a percolating porous medium (Adams et al., 2008; Dharmasiri et al., 2009). In this approach, cell paths through the pore space having a high torsion serve as micro centrifuges.

Most promising candidates for percolating porous media are open foams (sponges) and fleeces (fiber systems) widely used for particle filtering. Their structures are well investigated concerning porosity, specific surface area, tortuosity, percolation probability etc. These quantities are related to filtration properties such as flow rate, filtration efficiency and particle holding capability. Materials design of porous media for filtration processed is based on simulation of two-phase (liquid-solid) flow through a pore space including the interaction of solid particles with the inner surface (Peker and Helvaci, 2008). This allows for insights into the filtration process and involves the estimation of flow rates, filtration efficiencies, etc. depending on the permeability of the pore space.

Fig. 7 shows visualizations of $\mu \mathrm{CT}$ scans of the samples. The first one is from a fleece of nylon fibers with a mean width of about $20 \mu \mathrm{m}$, Fig. $7 \mathrm{a}$. Furthermore, as a reference specimen we investigate a (partially) open polyurethane foam with a mean number of about 70 pores per inch, Fig. $7 \mathrm{~b}$. The images were taken at the beamline ID19 of the European Synchrotron Radiation Facility in Grenoble. To achieve optimal contrast between the solid matter and the pore space, an inline phase contrast mode was applied under coherent radiation and a phase retrieval was involved into the tomographic reconstruction. All visualizations show cubic subimages consisting of $512^{3}$ pixels out of $2048^{3}$-data sets. The lateral resolution was $0.7 \mu \mathrm{m}$ for the fiber fleece and $1.1 \mu \mathrm{m}$ for the open foam.
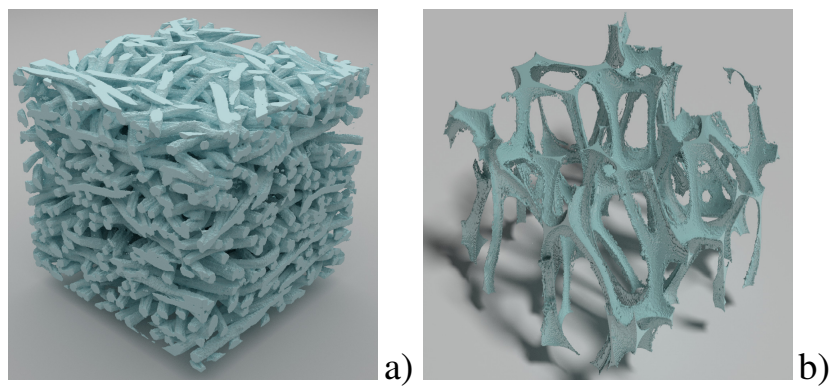

Fig. 7. Visualizations of $512^{3}$ out of $2048^{3}$ pixels of $\mu C T$-scans: (a) of a fiber fleece and (b) of a partially open foam.
For both cases, the direction of the fluid flow is parallel to the $z$-axis (in Fig. 7 from top to bottom). The open foam is nearly isotropic, while the distribution of the fiber system is invariant with respect to rotations around the $z$-axis, only. An open problem is the characterization of torsion of possible cells' trajectories in the pore space which influences the specific chromatographic interaction of target cells with inner surfaces. In the case of a high local torsion, a huge number of collision events of passing cells with the charged inner surface of the porous medium as well as a tight fit of interacting cells to the surface can be expected.

To extract discrete curves from 3D volume data, we are following the approach of Peyrega and Jeulin (2013) who computed geodesic distances based on a fast marching algorithm (Sethian, 1999), while valuable hints on the implementation of propagation algorithms are given in Baerentzen (2001). However, in particle-fluid two-phase flow through pore spaces the particles usually do not follow shortest paths. Thus, in order to be closer to real particle trajectories the above algorithm was modified in the following way: Taking into consideration that, in average, flow speed increases with increasing distance from the inner surface, the rate of faster particles passing the pore space is much higher than that of slower ones. Therefore, the typical particle is passing on a trajectory far from the inner surface. In order to simulate this effect, the Euclidean distance transform is performed on the pore space before extracting the curves. In the context of fluid flow simulation, the Euclidean distance is interpreted as speed and the particles paths are represented by discrete curves with shortest geodesic time (Soille, 1999).
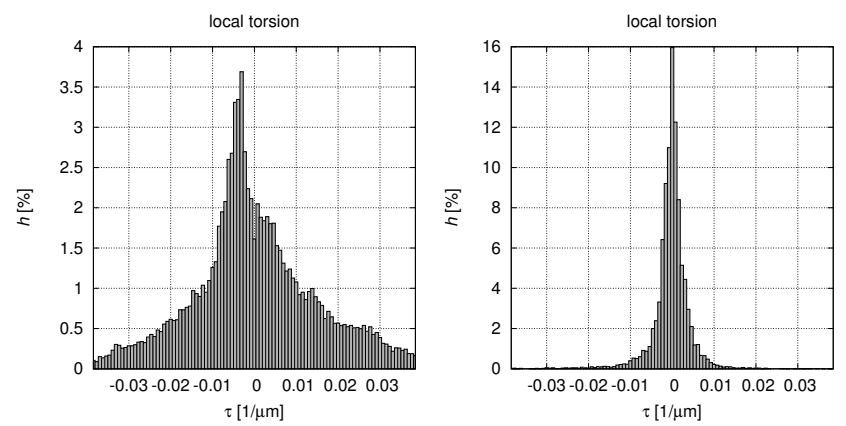

Fig. 8. Histograms $h$ of torsion estimation (left) of the fiber fleece and (right) of the partially open foam.

Generally, the distribution of the random porous medium influences the torsion distribution of paths through the pore space. Thus, it is not very surprising that the torsion distribution of the fiber system differs 
from that of the partially open foam (Fig. 8). First, we recognize that the variation of the torsion of the curves through the fiber system is much stronger than that for the foam. A reason for this is that the fiber system has a much lower porosity than the foam. On the other hand, one should take into consideration that for a (theoretical) system of parallel fibers with directions orthogonal to the flow direction, the torsion would be zero everywhere even in cases of low porosity. Thus, the variation of torsion depends on the fiber direction distribution as well. Furthermore, the torsion distribution is not symmetric (not invariant with respect to reflection at the origin). In Fig. 8 (left) one can observe a significant shift of the histogram to negative torsion, while that of the foam is nearly symmetric. This means that the fiber system is not invariant with respect to reflection at the xy-plane. Fluid flow from top to bottom is left rotating on average. This effect is caused by the melt-blown production process of the fiber system, where a lattice of fiber spinnerets is rotated. Even if the fibers are strongly swirled during their down-laying, the fleece inherits the rotation direction of the fiber spinnerets' lattice.

The consequences are twofold: 1. The torsion distribution is a quantity which allows for the detection of asymmetries of macroscopically homogeneous structures. 2. The torsion distribution of paths through the pore space of porous media can be influenced significantly by their production processes.

\section{DISCUSSION}

The discretization of space curves described above fundamentally differs from that usually investigated in discrete differential geometry, where a discrete curve of an arc length parameterized function $f$ is defined as the set of points $f\left(t_{i}\right)$ for equally spaced $t_{i}$. As pointed out e.g., in Carroll et al. (2014), this kind of discretization directly leads to a discrete version of Frenet's equations. Carroll et al. presented an approach of estimating curvature and torsion resulting from a discrete Frenet equation system which is free of any error term. However, the discretization of $f$ on which this approach is based differs from the outer Jordan discretization and, unfortunately, until now there is no way to extend the approach on Jordan discretization.

Clearly, Cesàro's mean is a typical low-pass filter applied in the frequency domain. Thus, one can expect that the use of any other low pass filter, such as binomial filters of higher order, would lead to similar errors of torsion estimation, where strong smoothing with large filter masks can be performed effectively via frequency domain, only. An advantage of Cesàro's mean over other low-pass filters is a more theoretical one that allows us to make use of Fejer's theorem which states that torsion estimation is multigrid convergent.

Other smoothing techniques, like fitting polynomials, the use of smoothing splines, and nonparametric techniques such as locally weighted scatter plot smoothing (LOWESS), etc., do not completely remove the high frequencies induced by sampling the curve on a lattice. However, even small amplitudes of high frequencies, which are not removed by smoothing, can have considerable impact on the third derivative used in the torsion estimation. In particular, third derivatives of smoothing cubic splines are not continuous, and hence, their application in torsion estimation cannot be recommended.

The way of extracting discrete curves based on shortest geodesic time is surely an empirical one, and the obtained curves are probably far from real particle paths. More realistic results could be obtained by two-phase fluid/solid-flow simulation or a direct observation of particle motion by in-situ experiments with $\mu \mathrm{CT}$. The last method was applied in Blankenburg et al. (submitted), where sequences of 3D images were taken from a partially open foam by $\mu \mathrm{CT}$, while simultaneously a particle suspension is pumped through the pore space. However, for realistic simulation studies one needs detailed information on the boundary condition at the inner surface of the porous media, and in particular the particlesurface interaction is not yet known. In the in-situ experiments made by Blankenburg et al. (submitted), a scanning rate of two images per second was reached which allows a tracking of very slow particles, only. Furthermore, the discrete particle paths extracted from the image sequences differ significantly from those investigated in this paper.

\section{ACKNOWLEDGMENT}

This work was supported by the German Federal Ministry of Education and Research (BMBF) under grant MNT/05M13RCA/AniS. The authors thank Alexander Rack for his valuable support during the imaging of the 3D structure of the porous materials at beamline ID19 of the European Synchrotron Radiation Facility, Grenoble.

\section{REFERENCES}

Adams AA, Okagbare P, Feng J, McCarley RL, Murphy MC, Soper SA (2008). Capture and enumeration of circulating tumor cells from peripheral blood using microfluids. J Am Chem Soc 130:8633-41. 
Bae CJ, Erdonmez CK, Halloran JW, Chiang YM (2013). Design of battery electrodes with dual-scale porosity to minimize tortuosity and maximize performance. Adv Mat 25:1254-8.

Baerentzen A (2001). On the implementation of the fast marching methods for 3D lattices. Tech Rep IMM-REP2001-13, Lyngby: Tech Univ Denmark.

Blankenburg C, Daul C, Ohser J (2015). Torsion of particle trajectories through pore space and its estimation using information on local pixel configurations. In: Pirard E, ed. Proc 14th Int Congr Stereol Image Anal (ICSIA). Liège: ISS.

Carroll D, Hankins E, Kose E, Sterling I (2014). A survey of the differential geometry of discrete curves. Math Intell $36: 28-35$.

Chen-Wiegart YK, DeMike R, Erdonmez C, Thornton K, Scott A. Barnett SA, Wang J (2014). Tortuosity characterization of $3 \mathrm{D}$ microstructure at nano-scale for energy storage and conversion materials. J Power Sources 249:349-56.

Coeurjolly D, Serge M, Laure T (2001). Discrete curvature based on osculating circles estimation. Lect Notes Comp Sci 2059:303-12.

Coeurjolly D, Svensson S (2003). Estimation of curvature along curves with application to fibres in 3D images of paper. Lect Notes Comp Sci 2749:247-54.

Coleman SY, Pritchett CJ (1990). Random rotations in simulation with computer $3 \mathrm{D}$ reconstruction. Acta Stereol 9:207-18.

Crenshaw HC, Ciampaglio CN, McHenry M (2000). Analysis of the three-dimensional trajectories of organisms: estimates of the velocity, curvature and torsion from positional information. $\mathrm{J}$ Exp Biol 203:961-82.

Dharmasiri U, Balamurugan S, McCarley RL, Spivak D, Soper SA (2009). Highly efficient capture and enumeration of low abundance prostate cancer cells using prostate-specific membrane antigen aptamers immobilized to a polymeric microfluidic device. Electrophoresis 30:3289-300.

Fullman RL (1953). Measurement of approximately cylindrical particles in opaque samples. Trans Metall AIME 197:1267-8.

Gaiselmann G, Manke I, Lehnert W, Schmidt V (2013). Extraction of curved fibers from 3D data. Image Anal Stereol 32:57-63.

Gommes CJ, Bons AJ, Blacher S, Dunsmuir JH, Tsou AH (2009). Practical methods for measuring the tortuosity of porous materials from binary or gray-tone tomographic reconstructions. AIChE J 55:2000-12.

Grisan E, Foracchia M, Ruggeri A (2003). A novel method for the automatic evaluation of retinal vessel tortuosity.
In: Proc 25 Int Conf IEEE Eng Med Bio Soc. 1:866-9.

Kehtarnavaz ND, de Figueiredo JP (1988). A 3D contour segmentation scheme based on curvature and torsion. IEEE T Pattern Anal 10:707-13.

Lewinger T, Gomes JD, Lopes H, Craizner M (2005). Curvature and torsion estimators based on parametric curve fitting. Comp Graph 29:641-55.

Medina R, Wahle A, Olszewski ME, Sonka M (2004). Curvature and torsion estimation for coronary-artery motion analysis. Medical Imaging 2004. Proc SPIE 5369:504-15.

Meng S, Costa LdF, Geyer SH, Viana MP, Reiter C, Müller GB, Weninger W (2008). Three-dimensional description and mathematical characterization of the parasellar internal carotid artery in human infants. J Anat 212:636-44.

Moeslang A, Pieritz RA, Boller B, Ferrero C (2009). Gas bubble network formation in irradiated beryllium pebbles monitored by X-ray microtomography. J Nucl Mat 386-388:1052-5.

Mokhtarian F (1997). A theory of multiscale, torsion-based shape representation for space curves. Comp Vis Image Und 68:1-19.

Nguyen TP, Debled-Rennesson I (2008). Curvature and torsion estimators from 3D curves. Advances in Visual Computing. Lect Notes Comp Sci 5358:688-99.

Nguyen TP, Debled-Rennesson I (2009). On local properties of digital curves. Int J Shape Modeling 14:105-25.

Ohser J, Ferrero C, Wirjadi O, Kuznetsova A, Düll J, Rack A (2012). Estimation of the probability of finite percolation in porous microstructures from tomographic images. Int J Mat Res 103:184-91.

Ohser J, Nagel W, Schladitz K (2009). Miles formulae for Boolean models observed on lattices. Image Anal Stereol 28:77-92.

Ohser J, Schladitz K (2009). 3D Images of Materials Structures - Processing and Analysis. Weinheim, Berlin: Wiley VCH.

Pao YC, Lu JT, L. RE (1992). Bending and twisting of an in vivo coronary artery at a bifurcation. $\mathrm{J}$ Biomech 25:287-95.

Patasius M, Marozas V, Lukosevicius A, Jegelevicius D (2007). Model based investigation of retinal vessel tortuosity as a function of blood pressure: preliminary results. In: Proc 29th Int Conf IEEE Eng Med Biol Soc. 6459-62

Peker SM, Helvaci SS (2008). Solid-Liquid Two-Phase Flow. Amsterdam: Elsevier.

Peyrega C, Jeulin D (2013). Estimation of tortuosity and reconstruction of geodesic paths in 3D. Image Anal Stereol 32:27-43. 
Pieritz RA, Reimann J, Ferrero C (2011). 3D tomography analysis of the inner structure of pebbles and pebble beds. Adv Eng Mat 13:145-55.

Puentes J, Garreau M, Lebreton H, Roux C (1998). Understanding coronary artery movement: a knowledge-based approach. Artif Intell Med 13:20737.

Rhines FN (1977). Microstructure properties in materials. Metall Trans A 8:127-33.

Scagliarini A (2011). Geometric properties of particle trajectories in turbulent flows. J Turbulence 12:1-12.

Schneider R (1993). Convex Bodies: The Brunn-Minkowski Theory. Cambridge: Encyclopedia of Mathematics and Its Application Vol. 44, Cambridge University Press.

Sethian JA (1999). Level Sets Methods and Fast Marching Methods: Evolving Interfaces in Computational Geometry, Fluid Mechanics, Computer Vision and Materials Science. Cambridge: Cambridge University Press.

Soille P (1999). Morphological Image Analysis. Berlin,
Heidelberg: Springer-Verlag.

Spivak M (1979). A Comprehensive Introduction to Differential Geometry, vol. II. Houston: Publish or Perish, Inc.

Strandmark P, Ulén J, Kahl F, Grady L (2013). Shortest paths with curvature and torsion. In: Proc IEEE Int Conf Comp Vision. 2024-31.

Wood NB, Zhao SZ, Zambanini A, Jackson M, Gedroyc W, Thom AA, Hughes AD, Xu XY (2006). Curvature and tortuosity of the superficial femoral artery: a possible risk factor for peripheral arterial disease. J Appl Physiol 101:1412-8.

Zhao D, Wang H, Xiang Y (2012). Asymptotic behaviors of the stress fields in the vicinity of dislocations and dislocation segments. Phil Mag 92:2351-74.

Zhenga S, Qib Y (2011). Motion estimation of 3D coronary vessel skeletons from X-ray angiographic sequences. Comput Med Imag Graph 35:353-64.

Zygmund A (1988). Trigonometric series (2nd ed.). Cambridge: Cambridge University Press. 